\title{
37th ANNUAL SASKATCHEWAN CHRISTMAS BIRD COUNT — 1978
}

Compiled by MARY I. HOUSTON, 863 University Drive, Saskatoon, Saskatchewan, S7N 0J8.

Storms and low temperatures during the Christmas season discouraged or prevented a number of bird counts from being taken. This year's total was down to 49 localities with 68 species plus 1 additional race. The additional race was the Oregon race of the Dark-eyed Junco. The commoner race - that seen on 8 counts - is the Slate-colored. There were 4 additional species seen during count period but not on count day.

This year 2 new species, a Swainson's Hawk at Webb-Swift Current and a Yellow-bellied Sapsucker at Saskatoon, plus one new race, the Audubon's Warbler (a race of the Yellow-rumped Warbler) at Fort Walsh, were recorded on count days and 2 new species, the Varied Thrush at Pierce Lake and the Common Snipe at Webb-Swift Current were added to the list of species seen during count period. This brings the 37-year total to 124 species plus 2 races seen on count days, with 8 additional species seen during count periods but not on count days.

The most unusual species was the melanistic buteo, seen at close range by Bob Peart at Webb about once a week since late fall. Although melanistic buteos are notoriously difficult to identify and although Swainson's is the least likely of them to stay this far north in winter, this bird was totally dark underneath, so that from all the references consulted, this must be a Swainson's.

This year again we extend congratulations to the very en- thusiastic group at Togo, spearheaded by Cusie and Wanda May. Cusie made a round trip of $640 \mathrm{~km}$ to haul $4300 \mathrm{lb}$. of sunflower seeds to be distributed to the feeders; Wanda organized and compiled the daily Christmas period observations at 7 of the feeders. There are another 11 established bird feeding stations on farms in the Togo area.

Detailed information on their feeder observations; more information on habitats in other areas, and dates of species seen in count period but not on count day are available in the files.

Since Wascana Lake was allowed to freeze this fall, most of the waterfowl migrated, leaving Regina with only 3 waterfowl species. We miss their interesting extended list.

No unusual trends in species abundance were evident this year except that Hoary Redpolls were seen in only 2 localities.

Table 1. CHRISTMAS, BIRD COUNT PARTICIPANTS.

BANGOR. Mrs. A. Thompson.

BATTLEFORD-NORTH BATTLEFORD. Pern Cordery (compiler), Heywood MacDonnell.

BIGGAR. Harvey Renaud, Wayne Renaud, Guy Wapple (compiler), Robert Wapple, Rodney Wapple.

BROADVIEW. David Chaskavich, Don Weidl (compiler).

CANORA. Alfred Tataryn (compiler), Jeffrey Tataryn, Vicki Tataryn. 
DALMENY. Brian Sperling, Gilbert Sperling, Lloyd Sperling (compiler), Mara Sperling.

DILKE. Margaret Belcher (compiler), Mr. and Mrs. S. R. Belcher.

DUPEROW-RUTHILDA. Maurice L'hoir, Wayne Renaud, Guy Wapple (compiler).

EASTEND (29). Mike Gollop (compiler), Dale and Sheila Guthormson, Wayne Harris, Jane Jenkins, Rick Jerema, Sheila Lamont, Wayne Renaud, Guy Wapple.

EASTEND (24). Henri Lebastard.

EMMA LAKE. Bob Godwin (compiler), Julie Godwin, Kim Godwin, Angele Mcllveen, Dave Mcllveen, Kathy Meeres, Dan Paulson, Alan R. Smith.

\section{ENDEAVOUR. Norman Harris.}

FORT QU'APPELLE. E. M. Callin (compiler), Ethel Cockwill, B. De Vries, June Dick, Selma Hiebert, Ron and Esther Hooper, Joseph Kralkay, Lois Lamontagne, Shaun Rowell, Dorothy Williams.

FORT WALSH. Charles Harper, Wayne Harris, Bob Kreba, Sheila Lamont, Bob Luterbach, Wayne Renaud (compiler), Guy Wapple.

GARDINER DAM. Don Renaud, Wayne Renaud, Guy Wapple (compiler).

GLAMIS-WISETON. G. L. Jones (compiler), Chester Walker.

GRENFELL. Elizabeth Hubbard (compiler), John Hubbard.

HUMBOLDT. Ed Brockmeyer.

INDIAN HEAD. Cec and Betty Ashmore, Marg Barclay, Peter and Therese Barrett, Yvonne Brown, Margo Beaulieu, Ernie and Hazel Buglass, Cam, Elizabeth, Shane and MacKinley Davidson, Richard Escott, Joyce Gibson, Joan Halford, Gordon Howe, Jerome, Marc and Sheldon Longeau, Roy and Rose McLaughlin, John MacRobert, David Munro, Doris
Pals, Lloyd and Muriel Peterson, Adam, Gertie, Joan and Lorne Scott (compiler), Doug Simpson, Fred, Mary and Ken Skinner, Andrea, Dennis, Donna, Orland and Ron Thompson, Denis Williamson, John and Reta Winter, Bob Young.

KELVINGTON. Chet Sloan, Dianne Sloan (compiler), Marguerite Sloan.

KENASTON. P. Lawrence Beckie.

LACADENA. Rod Sheasby.

LANGHAM. Vi and Vic Harper.

LEADER. Daisy D. Meyers.

LOON LAKE. Ronald Hooper.

LUSELAND. Kerry Finley (compiler), Kim Finley, Estelle Martfeld.

MACDOWALL. Dan Neves, John Shadick, Stan Shadick (compiler).

MOOSE JAW. Achille, Beatrice, Catherine and Jean-Pierre Chabot, Carl Ellis, Ruth Hilling, Pat Kern, John Kern, Leith Knight (compiler), Cy Knight, Doug Francis, Jean Thomson, Molly Ritchie, Wilma Young.

MOOSE MOUNTAIN PARK. Autumn Downey, Jim Hines (compiler).

PIERCE LAKE. John E. Christie.

PRIMROSE LAKE. John E. Christie.

PRINCE ALBERT. Wayne Harris (compiler), Sheila Lamont, Margaret Lewis, Hertha, Todd and Tracie McCorriston, Richard Miller.

PRINCE ALBERT NATIONAL PARK. Ray Breneman, Suzanne Henry (compiler), David Henry, Michael Jones, Merv Syroteuk, Bruce Wilson, Charlie Zinkan.

RAYMORE. Greta Harris, Wayne Harris (compiler), Sheila Lamont.

REGINA. Gary Anweiler, Margaret Belcher, John and Tom Beveridge, $\mathrm{K}$. Costain, Kathleen Donauar, Robyn Donison, Mr. and Mrs. Murray Fines, Randall Fines, Valerie Harrison, Jim Hines (compiler), Jim and Shirley Jowsey, Ernest Kassian, Darlene 
auk, Greg Kraetzig, Bob Kreba, Eric nd Tony Lang, George Ledingham, hristine MacDonald, Joe Roberts, Rumens, Bill Russon, Allan and ianne Smith, Frank, Ian and May witzer, Christopher and Janie Vilhelm.

OUND LAKE. Doug Francis (comiler), Mr. and Mrs. Joe Grimeau.

ASKATOON. Mark Abley, Bernie nderson, Juhachi Asai, Jim Bardlell, Bob Besant, Garth Besant, Jeff esant, Joyce Besant, Ray Bisha, ligel Caulkett, Ian Creurer, John Edtrom, Arthur, Edward, Glen, Hartley nd Lawrence Fredeen, J. B. and M. - Gollop, Bruce Hanbidge, John lanbidge, Stuart Houston (compiler), ave, Don and Mary Houston, Tony ohns, Regina McDowell, Don 1cRobbie, Jo McRobbie, Sean Morrissey, Betty Mundy, Jim Mundy, Jan Neves, Lynn Oliphant, J. B. 'Neil, Pat O'Neil, John Polson, Don Renaud, Wayne Renaud, Adam Schhidt, John Shadick, Stan Shadick, im Slimmon, Alan Smith, Edward mith, Laura Smith, Tom Smith, Mary trickland, Judy Taylor, Guy Wapple.

KUULL CREEK. J. Bennetto (comiler), A. Drever, R. Flaig, D. Mann, M. Mann, R. Mann, T. Stevens.

OMME. David Black, Donald looper, Leila Hooper, Ronald looper (compiler).

PIRIT LAKE. Bill and Joyce Anaka compiler).

PRING VALLEY. Flossie Bogdan compiler), Larry and Nick Bogdan.

QUAW RAPIDS. Wayne Harris compiler), Sheila Lamont, Wayne enaud, Guy Wapple.

OGO. Phil and Jean Hern, Vic and Iga Hilderman, Walter and Ethel rupp, Cusie and Wanda May (comiler), Dick and Mary Smith, Howard nd Donelda Wilson, Orville and laine Wilson.

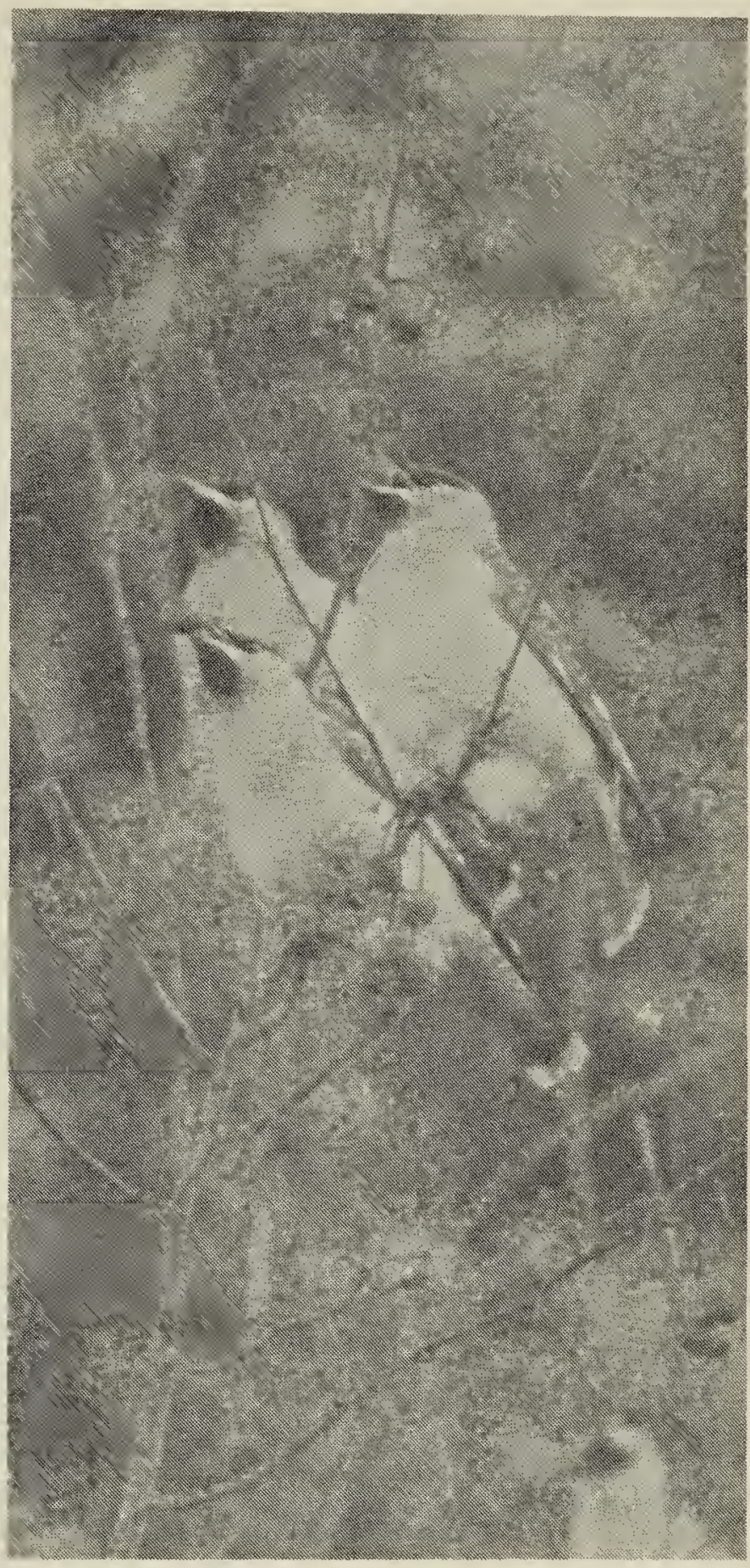

Bohemian Waxwings.

Wayne Harris

WAUCHOPE. Dale Hjertaas (compiler), Paule Hjertaas.

WEBB-SWIFT CURRENT. Bob Peart (compiler), Dave Short.

WHITE BEAR. Sig Jordheim.

WHITEBEECH. Ida and Lindsay Wotherspoon (compiler).

WOLSELEY. J. Donald Hayward.

YORKTON. Margaret Bromley, Bernard and Penny Laarueld, Avery and Jean Matlock, Phil Pawluck (compiler), Mrs. Rosene Yanke. 
Table 2. CHRISTMAS COUNT METHODS OF OBSERVATION, WEATHEF AND TOTAL BIRDS.

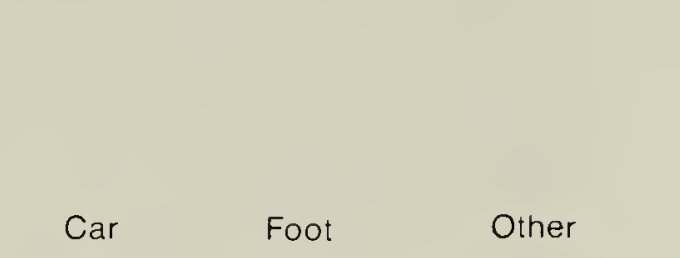

KM HR KM HR KM HR HR $\begin{gathered}\text { Temp. } \\ C\end{gathered}$ Dir KM/H CM $\quad$ Sky

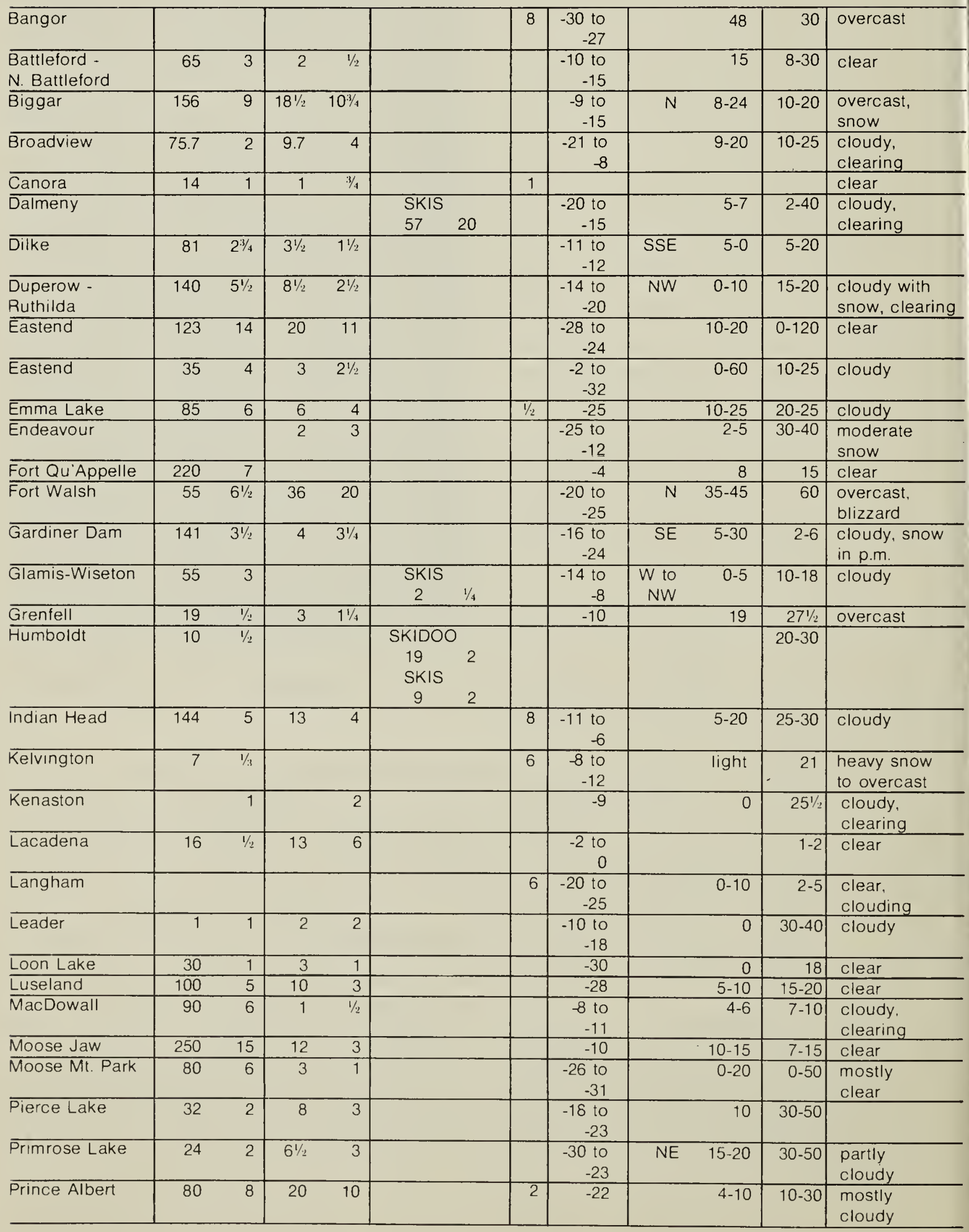


Table 2. CHRISTMAS COUNT METHODS OF OBSERVATION, WEATHER AND TOTAL BIRDS.

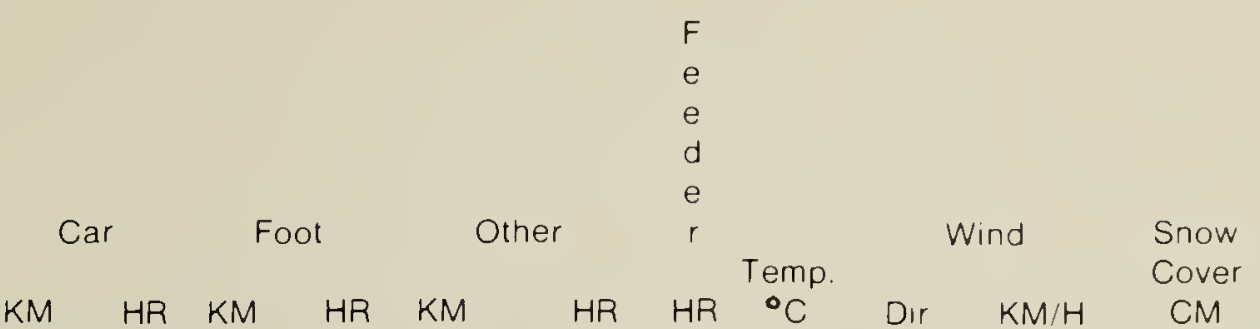

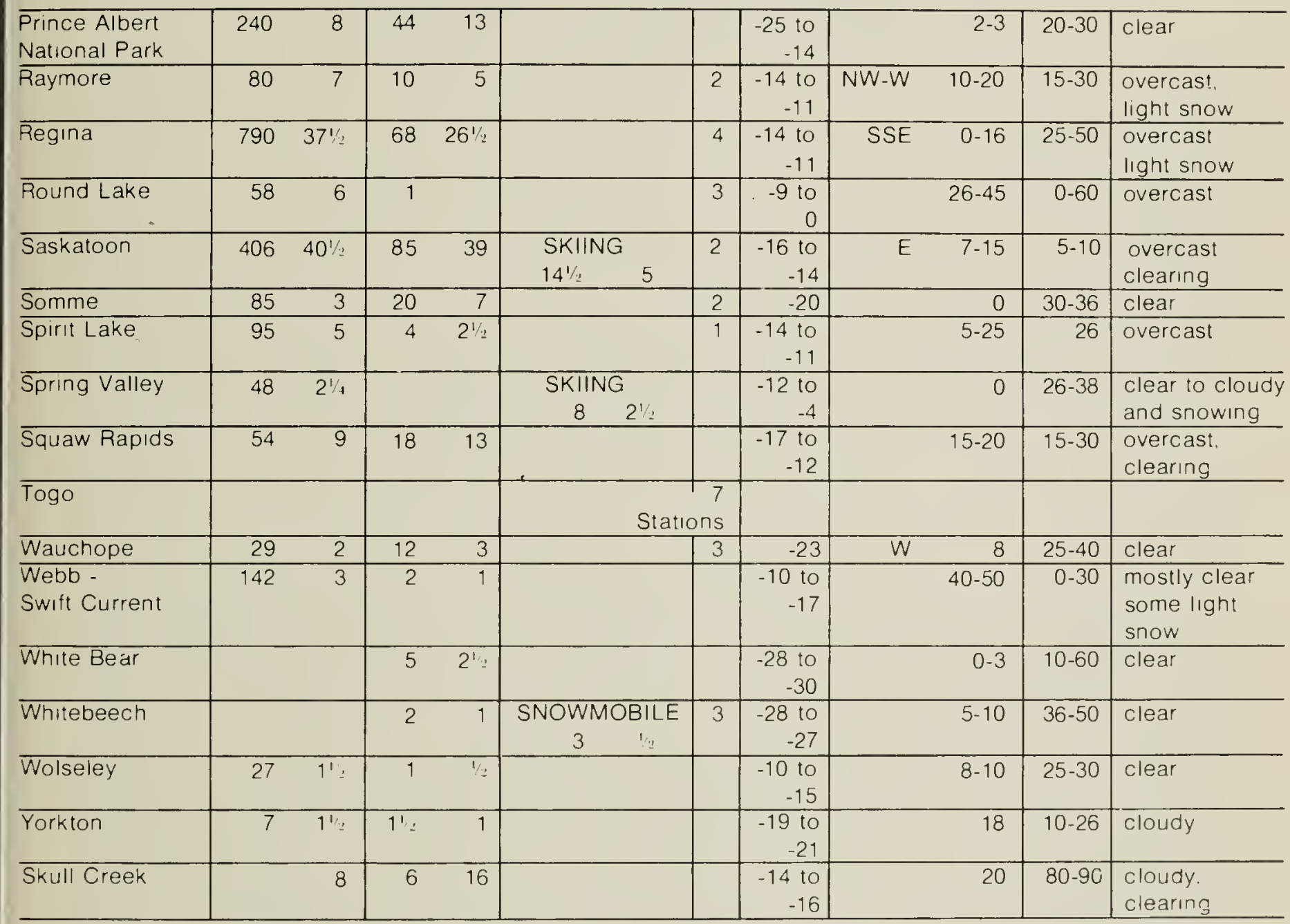


Table 3. BIRDS RECORDED FROM MORE THAN ONE LOCALITY ON COUNT DAY AND IN COUNT PERIOD (+).

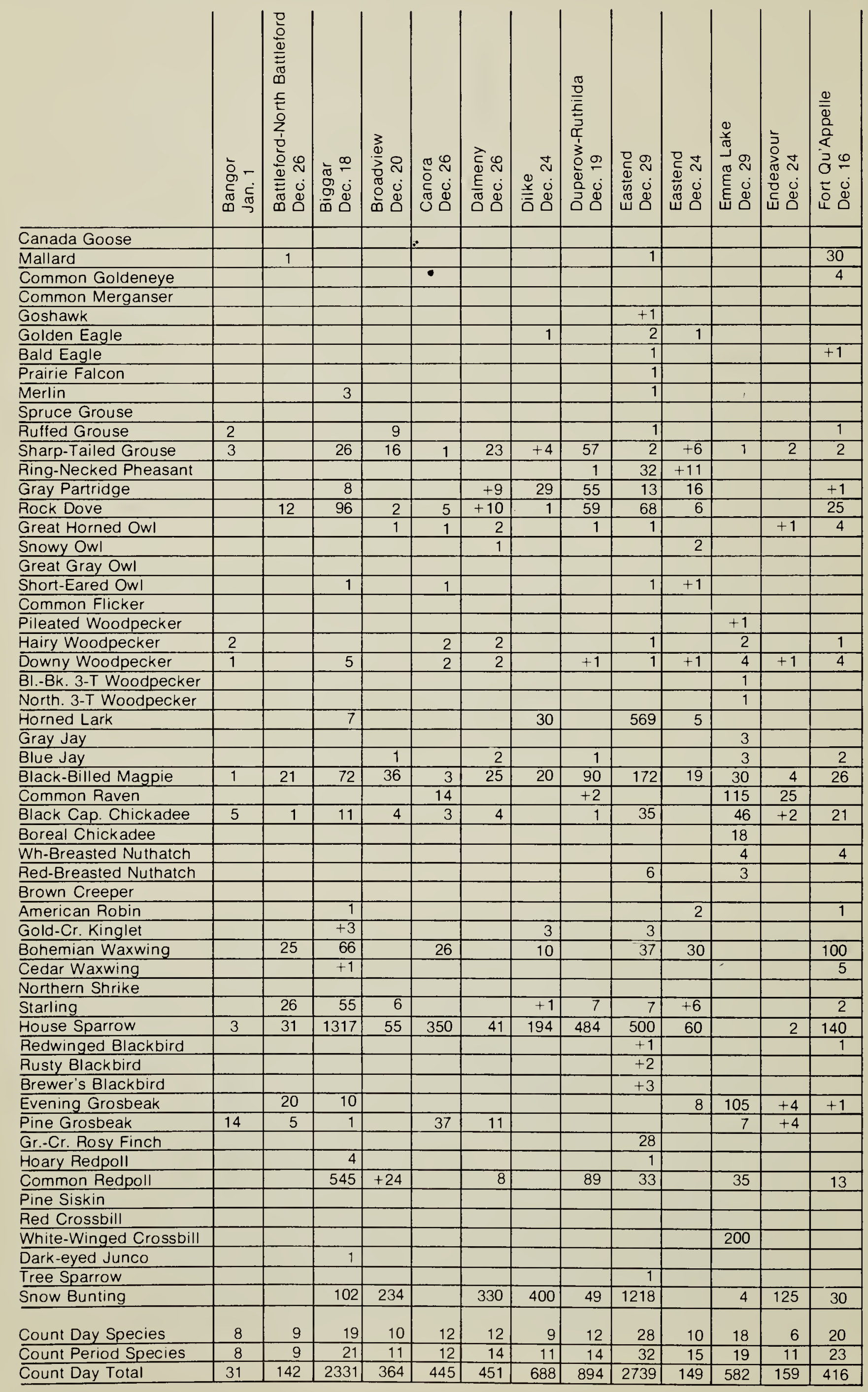




\section{Table 3. BIRDS RECORDED FROM MORE THAN ONE LOCALITY ON COUNT DAY AND IN COUNT PERIOD (+).}

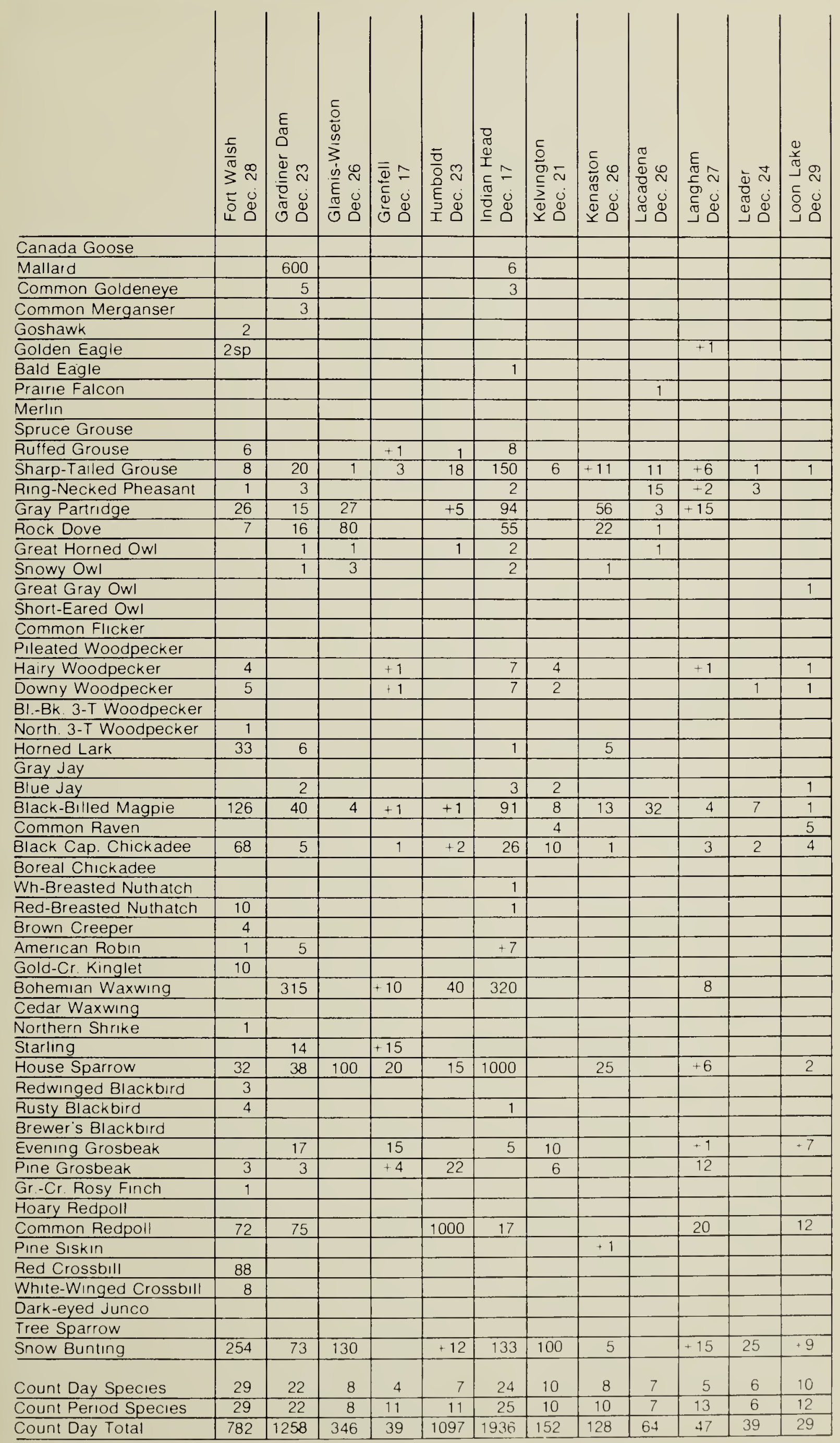


Table 3. BIRDS RECORDED FROM MORE THAN ONE LOCALITY ON COUNT DAY AND IN COUNT PERIOD (+).

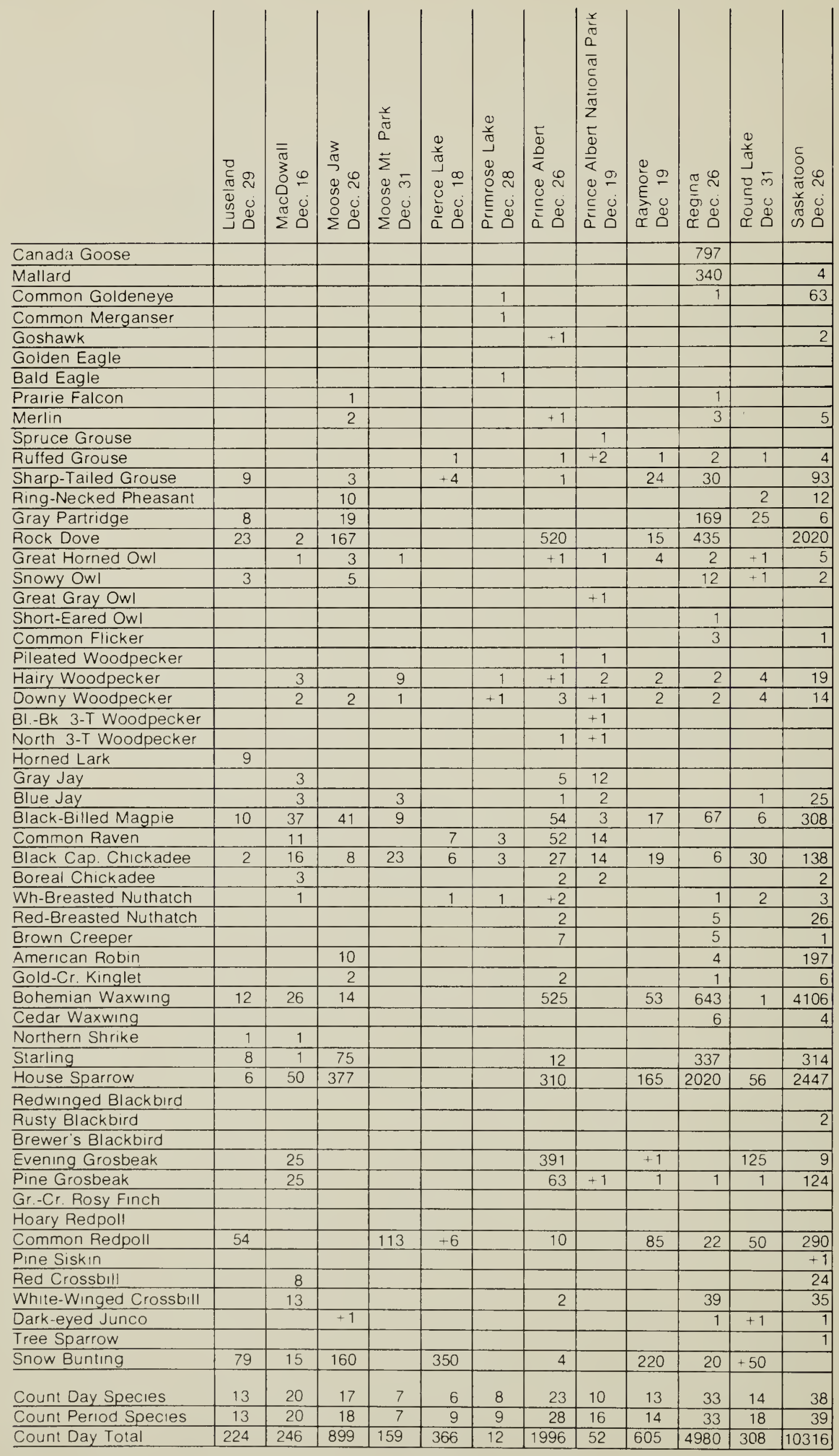


Table 3. BIRDS RECORDED FROM MORE THAN ONE LOCALITY ON COUNT DAY AND IN COUNT PERIOD (+).

\begin{tabular}{|c|c|c|c|c|c|c|c|c|c|c|c|c|}
\hline & 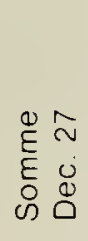 & 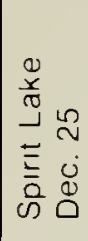 & 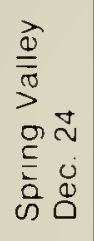 & 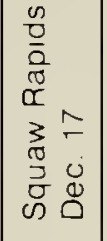 & \begin{tabular}{ll}
$\circ$ & - \\
$\circ$ & \multicolumn{1}{c}{} \\
$\circ$ & 0 \\
& 0
\end{tabular} & 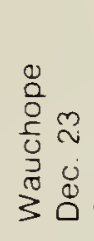 & 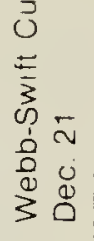 & 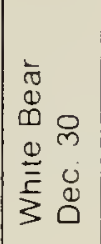 & 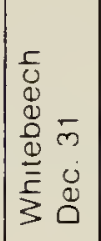 & $\mid \begin{array}{ll}\frac{\partial}{0} & m \\
\frac{0}{0} & v \\
\frac{w}{2} & 0 \\
3 & 0\end{array}$ & 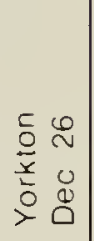 & 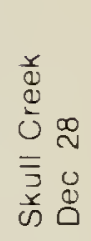 \\
\hline Canada Goose & & & & & & & +1 & & & & & \\
\hline Mallard & & & & 24 & & & & & & & & \\
\hline Common Goldeneye & & & & 135 & & & & & & & & \\
\hline Common Merganser & & & & 2 & & & & & & & & \\
\hline Goshawk & 1 & & & 3 & 1 & & & & & & & \\
\hline Golden Ëagle & & & +1 & & & & +1 & +2 & & & & +1 \\
\hline Bald Eagle & & & & 15 & & & & & & & & +1 \\
\hline Prairie Falcon & & & & & & & & +1 & & & & +1 \\
\hline \multicolumn{13}{|l|}{ Merlın } \\
\hline Spruce Grouse & & & & 1 & & & & & & & & \\
\hline Ruffed Grouse & 6 & 1 & & 8 & 4 & 6 & & & & 2 & & \\
\hline Sharp-Tailed Grouse & +4 & 11 & 8 & & $\overline{5}$ & 6 & 51 & 1 & +1 & 8 & 10 & 18 \\
\hline Ring-Necked Pheasant & & & & & & & & 15 & & & & 23 \\
\hline Gray Partrıdge & 9 & 20 & 42 & & +8 & 3 & 12 & 35 & & +7 & 12 & 8 \\
\hline Rock Dove & +6 & & 24 & & & 11 & 64 & 50 & & & & \\
\hline Great Horned Owl & 1 & 1 & +1 & 1 & 1 & 2 & & & & +1 & 1 & 7 \\
\hline Snowy Owl & & & 1 & & +1 & & +1 & & & +1 & & +1 \\
\hline \multicolumn{13}{|l|}{ Great Gray Owl } \\
\hline Short-Eared OwI & & & & & & & +1 & & & & & \\
\hline Common Flıcker & & & & & & & +1 & & & & & \\
\hline Plleated Woodpecker & & & & & +1 & & & & & & & \\
\hline Hairy Woodpecker & 7 & 8 & & 7 & 3 & 2 & & & 2 & 3 & & 2 \\
\hline Downy Woodpecker & 1 & 9 & & 2 & 2 & 1 & +1 & +1 & 3 & 1 & 1 & 3 \\
\hline Bl.-Bk. 3-T Woodpecker & & & & 2 & & & & & & & & \\
\hline North 3-T Woodpecker & 3 & & & 2 & & & & & & & & \\
\hline Horned Lark & & +2 & 28 & & & & 16 & +5 & & 2 & & 186 \\
\hline Gray Jay & 1 & & & 5 & & & & & 1 & & & \\
\hline Blue Jay & 4 & 1 & & 6 & 2 & & & & & & & 1 \\
\hline Black-Billed Magpie & 16 & 26 & 7 & 11 & 2 & 13 & 14 & 29 & +1 & 8 & 15 & 48 \\
\hline Common Raven & 30 & +1 & & 60 & 2 & & & & 2 & & & \\
\hline Black Cap. Chıckadee & 10 & 26 & & 20 & 30 & 17 & +1 & +1 & 6 & 2 & & 52 \\
\hline Boreal Chickadee & & & & 38 & 4 & & & & & & & \\
\hline Wh-Breasted Nuthatch & & 2 & & & 1 & & & & & & & \\
\hline Red-Breasted Nuthatch & & & & 23 & & & & & & & & \\
\hline \multicolumn{13}{|l|}{ Brown Creeper } \\
\hline American Robin & & & & & & & +1 & & & & & +1 \\
\hline \multicolumn{13}{|l|}{ Gold-Cr. Kinglet } \\
\hline Bohemian Waxwing & +2 & 13 & +14 & & 1 & & & 28 & & & 9 & 47 \\
\hline \multicolumn{13}{|l|}{ Cedar Waxwing } \\
\hline Northern Shrike & +1 & & & 1 & & & & & & & & \\
\hline Starlıng & & 2 & & & & 4 & & 3 & & & & \\
\hline House Sparrow & 68 & 276 & 280 & & 58 & 310 & 23 & 100 & +25 & 50 & 41 & 75 \\
\hline \multicolumn{13}{|l|}{ Redwinged Blackbird } \\
\hline Rusty Blackbird & & & +1 & & & & & & & & & 1 \\
\hline Brewer's Blackbırd & & & & & & & & & & +1 & & \\
\hline Evening Grosbeak & 1 & +20 & & 2 & 322 & 15 & & & 15 & & & \\
\hline PIne Grosbeak & 14 & 7 & & 66 & +6 & & & & +3 & +3 & .15 & \\
\hline \multicolumn{13}{|l|}{ Gr.-Cr. Rosy Finch } \\
\hline \multicolumn{13}{|l|}{ Hoary Redpoll } \\
\hline Common Redpoll & +2 & 55 & & 63 & 4 & 8 & +7 & & & 8 & 11 & \\
\hline PIne Sıskın & & +2 & & & & & & & & & & \\
\hline \multicolumn{13}{|l|}{ Red Crossbill } \\
\hline White-Winged Crossbill & & & & 93 & & & & & & & & \\
\hline Dark-eyed Junco & 1 & & & & & 1 & +8 & & & & & \\
\hline Tree Sparrow & & & & & & 1 & & & & & & \\
\hline Snow Bunting & 26 & 2 & 1200 & 10012 & +8 & +2 & 900 & +200 & & 50 & .8 & 3000 \\
\hline Count Day Species & 17 & 16 & 8 & 27 & 16 & 15 & 8 & 8 & 6 & 10 & 8 & 14 \\
\hline Count Period Species & 22 & 20 & 12 & 27 & 21 & 16 & 19 & 14 & 10 & 15 & 10 & 19 \\
\hline Count Day Total & 198 & 460 & 1590 & 10608 & 442 & 401 & 1081 & 261 & 29 & 134 & 100 & 347 \\
\hline
\end{tabular}




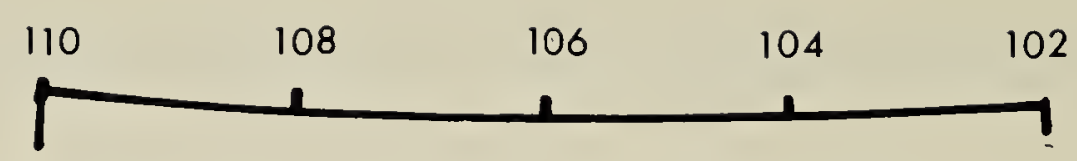

1. BANGOR

2. BATTLEFORD-NORTH BATTLEFORD

3. BIGGAR

4. BROADVIEW

5. CANORA

6. DALMENY

7. DILKE

8. DUPEROW-RUTHILDA

9. EASTEND

10. EASTEND

11. EMMA LAKE

12. ENDEAVOUR

13. FORT QU'APPELLE

14. FORT WALSH

15. GARDINER DAM

16. GLAMIS-WISETON

17. GRENFELL

18. HUMBOLDT

19. INDIAN HEAD

20. KELVINGTON

21. KENASTON

22. LACADENA

23. LANGHAM

24. LEADER

25. LOON LAKE
26. LUSELAND

27. MACDOWALL

28. MOOSE JAW

29. MOOSE MOUNTAIN PARK

30. PIERCE LAKE

31. PRIMROSE LAKE

32. PRINCE ALBERT

33. PRINCE ALBERT NATIONAL PARK

34. RAYMORE

35. REGINA

36. ROUND LAKE

37. SASKATOON

38. SOMME

39. SPIRIT LAKE

40. SPRING VALLEY

41. SQUAW RAPIDS

42. TOGO

43. WAUCHOPE

44. WEBB-SWIFT CURRENT

45. WHITE BEAR

46. WHITEBEECH

47. WOLSELEY

48. YORKTON

49. SKULL CREEK

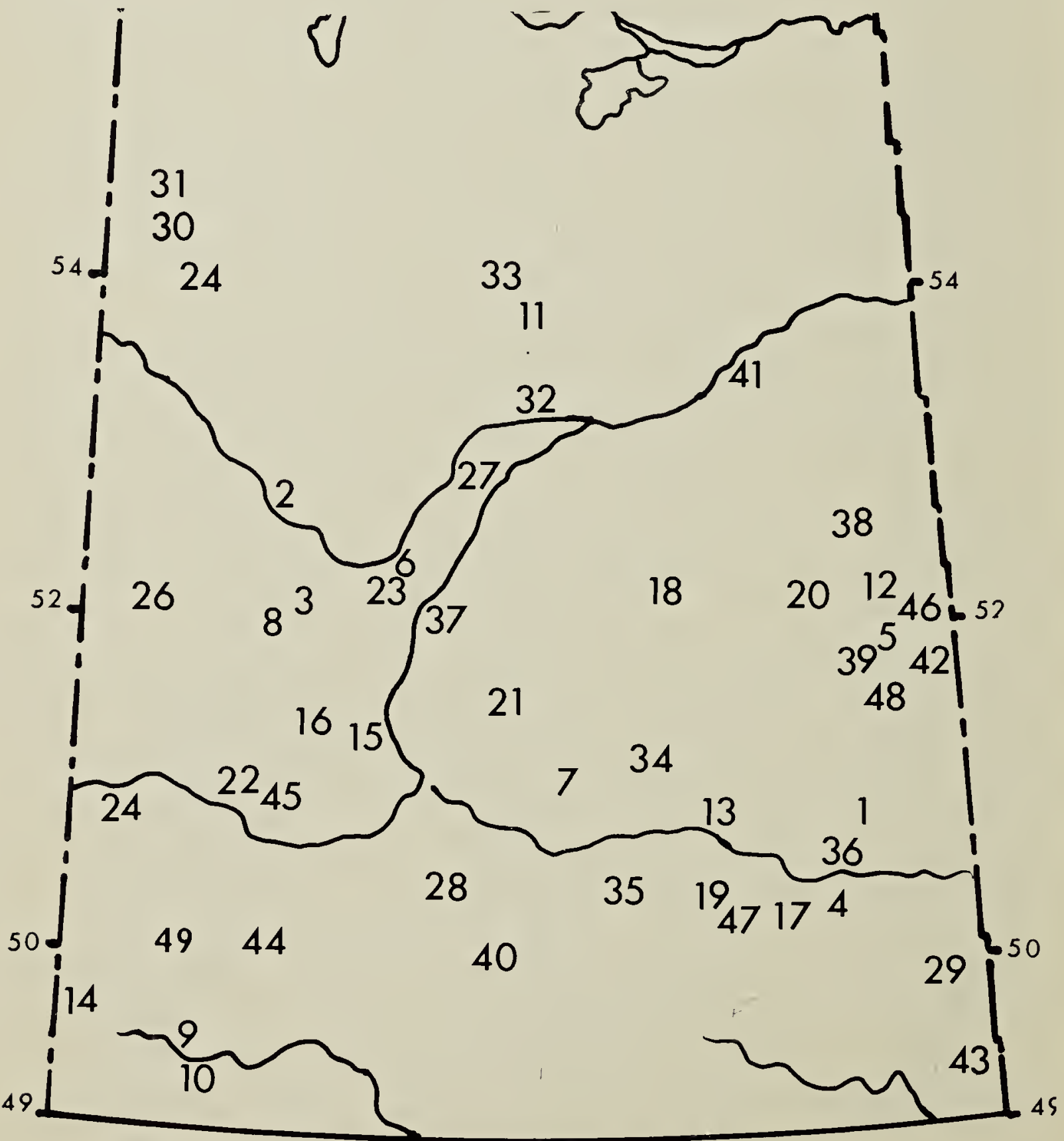




\section{Table 4. SPECIES RECORDED FROM ONLY ONE LOCALITY}

$\begin{aligned} \text { No. } & \text { Species } \\ 1 & \text { Pintail } \\ 3 & \text { Lesser Scaup } \\ 1 & \text { Sharp-shinned Hawk } \\ 1 & \text { Swainson's Hawk } \\ 1 & \text { Gyrfalcon } \\ 1 & \text { Willow Ptarmigan } \\ +1 & \text { Common Snipe } \\ 1 & \text { Hawk Owl } \\ 1 & \text { Long-eared Owl } \\ 1 & \text { Saw-whet Owl } \\ 1 & \text { Yellow-bellied Sapsucker } \\ 1 & \text { Common Crow } \\ +1 & \text { Varied Thrush } \\ 1 & \text { Townsend's Solitaire } \\ 1 & \text { Yellow-rumped (Audubon's) Warbler } \\ 1 & \text { Dark-eyed (Oregon) Junco } \\ 3 & \text { Lapland Longspur }\end{aligned}$

\section{NORTHWEST}

\section{TERRITORIES}

\section{CHRISTMAS BIRD} COUNTS

\section{FORT SMITH}

Date: Dec. 26, 1978.

Weather: clear with patches of ice fog; temp. $-35^{\circ} \mathrm{C}$; calm; snow $25 \mathrm{~cm}$. Routes Covered: Fort Smith, west along Highway 5 to the Fox Holes Road intersection; south along the Pine Lake Road to Salt River; east along the Hay Camp Road to Fort Fitzgerald. $150 \mathrm{~km}$ in $3 \frac{1}{2}$ hours.

Birds seen: Goshawk, 1; Gyrfalcon, 1; Willow Ptarmigan, 3; Great Gray Owl, 1; Gray Jay, 3; Common Raven, 141; Boreal Chickadee, 1; Pine Grosbeak, 35; Redpoll sp. 30. 8 Species, 215 individuals. (Add: Evening Grosbeak, 8, Dec. 23).

Contributors: Ralph Checkley, Nancy and Ian Church, Denys Cook, Dan Graham (compiler), Grant Hogg, Martha Jalkotzy, Carl and Jan Lentowicz, Betty Montgrand, C. Schaefer, Ray
Locality

Gardiner Dam

Squaw Rapids

Saskatoon

Webb-Swift Current

Fort Walsh

Primrose Lake

Webb-Swift Current

Squaw Rapids

Regina

Endeavour

Saskatoon

Regina

Pierce Lake

Saskatoon

Fort Walsh

Wauchope

Eastend
Map No.

15

41

37

44

14

31

44

41

35

12

37

35

30

37

14

43

9

Whaley. (Food and hot drink prepared by Linda Graham!)

\section{YELLOWKNIFE}

Date: Dec. 17, 1978.

Weather: temp. $-32^{\circ} \mathrm{C}$; wind SE 8 $\mathrm{km} / \mathrm{hr}$; clear.

Coverage: $73 \mathrm{~km}$ by car and $36 \mathrm{~km}$ on foot in 10.25 hours.

Birds seen: Spruce Grouse, 6; Willow Ptarmigan, 31; Gray Jay, 1; Common Raven, 234; Boreal Chickadee, 1; Starling, 2; House Sparrow, 29; Hoary Redpoll, 10; Redpoll sp. 128; Snow Bunting, 1. 10 species, 443 individuals.

Contributors: Jenny Clark, Judy Donaldson, George Gibson. Troy Gibson, Don Karasiuk, Cliff Matthews (compiler), Gail McCorrister. Dave Sutherland, Wendy Thompson. 